\title{
CPAP after endoscopic procedures as add-on therapy for the treatment of tracheal stenosis: a case series
}

\author{
Adriano Gesuele ${ }^{1}$, Simone Gambazza ${ }^{2,3}$, Marta Lazzeri ${ }^{4}$, Serena Conforti ${ }^{4}$ \\ ${ }^{1}$ First Level Specializing Master in Physiotherapy and Respiratory Rehabilitation, University of Milan; ${ }^{2}$ UOC Direzione \\ delle Professioni Sanitarie, Fondazione IRCCS Ca' Granda Ospedale Maggiore Policlinico, Milan; ${ }^{3}$ Department of \\ Clinical Sciences and Community Health, University of Milan; ${ }^{4}$ Department of Cardiothoracic and Vascular Surgery, \\ ASST Grande Ospedale Metropolitano Niguarda, Milan, Italy
}

\begin{abstract}
Tracheal stenosis represents a possible complication in intubated or tracheotomised patients. Tracheal resection is currently the gold standard for the treatment of complex stenosis while granulomas and simple stenosis (e.g., web-like) are often treated by endoscopic procedures, which do not consistently give satisfactory long-term results, due to frequent relapses. Administering continuous positive airway pressure (CPAP) after endoscopic procedures might represent a new add-on option for the treatment of this complication. In this case series are presented two patients with tracheal stenosis showed after the removal of tracheostomy tube, both treated with CPAP. The results were straightforward: CPAP treatment helped to keep stable the tracheal lumen, without adverse effects. No further endoscopic dilations were necessary thereafter, with a likely positive impact on patients' quality of life and on health expenditure.
\end{abstract}

Simone Gambazza, U.O.C. Direzione delle Professioni Sanitarie, Fondazione IRCCS Ca' Granda Ospedale Maggiore Policlinico, Via Francesco Sforza 28, 20122 Milano, Italy.

E-mail: simone.gambazza@policlinico.mi.it

Key words: Continuous positive airway pressure; tracheal stenosis; endoscopy.

Contributions: AG, SG, ML, study concept and design; AG, ML, SC, data acquisition; AG, SG, ML, SC, data analysis and interpretation. All the authors have drafted the manuscript and reviewed the work critically, have read and approved the final manuscript and agreed to be accountable for all aspects of the work.

Disclosures: SG reports personal fees from Vertex Pharmaceuticals and Mylan, outside the submitted work.

Received for publication: 25 June 2019.

Accepted for publication: 11 October 2019.

${ }^{\circ}$ Copyright: the Author(s), 2019

Licensee PAGEPress, Italy

Monaldi Archives for Chest Disease 2019; 89:1121

doi: 10.4081/monaldi.2019.1121

This article is distributed under the terms of the Creative Commons Attribution Noncommercial License (by-nc 4.0) which permits any noncommercial use, distribution, and reproduction in any medium, provided the original author(s) and source are credited.

\section{Introduction}

Endotracheal intubation and tracheotomy represent two important therapeutic options in the management of respiratory failure. However, these procedures are not without complications and tracheal stenosis is the most frequent consequence [1]. This consists in the abnormal reduction of the diameter of the endotracheal lumen, which can even result in breathing difficulties as the airway get narrower. Tracheal stenosis is usually described together with cough, dyspnea, tirage, stridor, wheezing and cyanosis.

Incidence varies depending on the therapeutic choice used. According to some prospective studies, $3 \%$ of patients develop tracheal stenosis as a late post-extubation complication while 1.7$5.9 \%$ if tracheotomized [2]. Also, this event depends on the duration of the intubation and on the type of cuff used [1]. In recent years, the introduction of so-called high-volume-low-pressure cuffs, which adapt better to the shape of the endotracheal lumen, has considerably reduced the incidence of tracheal stenosis, mainly by reducing the pressure exerted on the mucosal walls [1].

Treatment of tracheal stenosis can be surgical or endoscopic. Among these therapeutic options, continuous positive airway pressure (CPAP) therapy after endoscopic dilatation has recently been reported, although few cases are described in the literature [3]. We will illustrate the application of CPAP after endoscopic dilatation procedure in two patients with diagnosis of tracheal stenosis following invasive ventilation.

\section{Case Reports}

\section{Case \#1}

A 35-year-old woman presented acute myopericarditis with rapid and progressive hepatorenal failure, hemodynamic impairment and development of cardiogenic shock. A percutaneous tracheotomy was planned on day eighteenth due to difficult and prolonged respiratory weaning [4]. From the first days of intensive care, the patient developed a flaccid tetraparesis for severe critical illness polyneuromyopathy, as documented by the presence of myoglobin in the urine and confirmed with the EMG examination performed on the $54^{\text {th }}$ day of hospitalization. On the $56^{\text {th }}$ day the cannula was removed, given the presence of effective reflexes of airway protection and the recovery of sufficient respiratory autonomy. The patient left the intensive care unit (ICU) on the $63^{\text {rd }}$ day.

After 18 days from the removal of the cannula, she presented shortness of breath, a prolonged inspiratory time with stridor and 
use of accessory respiratory muscles. The chest computed tomography $(\mathrm{CT})$ showed a trachea in axis, with a $3-3.5 \mathrm{~cm}$ caliber reduction below the glottal plane, extended for about $4 \mathrm{~cm}$, with a minimum tracheal air lumen of $6 \times 6 \mathrm{~mm}$. The bronchoscopy (performed on the $74^{\text {th }}$ day) confirmed the presence of a tracheal stricture at about $15 \mathrm{~mm}$ from the cordal plane, which extended for about $20 \mathrm{~mm}$. The trachea showed a tortuous course with a residual diameter of $5 \mathrm{~mm}$ (Grade III - Myers-Cotton subglottic stenosis classification scale). Therefore, it was scheduled an endoscopic laser surgery followed by dilatation with balloon (Figure 1), which was performed on the $77^{\text {th }}$ day and determined a discrete tracheal canalization.

After 91 days of hospitalization, the patient was discharged from the hospital and transferred to a rehabilitation center in good hemodynamic conditions, with adequate respiratory gas exchange, initial but incomplete recovery of diuresis (still required threeweek dialysis sessions) and a partial recovery of muscle strength.

Ten days after discharge, the patient underwent a follow-up bronchoscopy that confirmed the good trachea patency; however, the bronchoscopy performed 32 days later showed tracheal stricture recurrence. The patient was hospitalized again in order to perform a second tracheal dilatation by rigid tracheoscopy. The tracheal lumen was reduced by $2 \mathrm{~mm}$, therefore it was treated with radial incisions with papillotomy and subsequent balloon dilatation $(14 \times 40 \mathrm{~mm})$. At the end of the procedure, the recanalization was effective and persistent at the bronchoscopy performed three days apart. Nevertheless, tracheal stricture reoccurred, with the need for a third dilation after two weeks. From this moment on, the patient underwent periodic bronchoscopy follow-up, which were followed by endoscopic tracheal dilatations every time the stenosis occurred. In order to maintain the caliber achieved after endoscopic dilation, a tracheal stent was also used (Figure 2). The procedure was repeated two times (January 2014 and April 2014) with little success due to repeated displacements; therefore, it was definitively removed in May 2014. Over a period of more than two years, the patient performed 22 fiberoptic bronchoscopies and 14 endoscopic dilatations (Figure 3), which required anesthesia and the use of an operating room.

In April 2015, following the last endoscopic dilatation, there remained a double re-stenosis and malacia of the trachea as a result of multiple dilations. It was necessary to proceed with another dilatation (precut sphincterotomy) and insertion of rigid dilators with increasing caliber and balloon $(14 \times 40 \mathrm{~mm})$. At the end of the procedure, the canalization allowed the passage of a rigid $10 \mathrm{~mm}$ tracheoscope.

Considering the several recurrences of the double stenosis, the concomitant malacia and the repeated surgical procedures on the trachea, the patient was adapted to CPAP the day after the last endoscopic dilatation (April 2015), using a single-tube home ventilator (Breas ${ }^{\circledR}$ iSleep 20) with integrated humidifier and oralnasal interface (Resmed Mirage Quattro size M). Positive endexpiratory pressure (PEEP) was initially set to $12 \mathrm{cmH}_{2} \mathrm{O}$ and then reduced to $10 \mathrm{cmH}_{2} \mathrm{O}$ as the patient reported aerophagy, with objective confirmation of gastric distension. Indications were given to maintain the application of continuous positive pressure for all night long and at least two hours in the afternoon. Flexible bronchoscopy, performed after two and a half months (July 2015), confirmed that the trachea was discreetly channeled into the two stenotic tracts; the malacia persisted when it coughed, but on the other hand shortness of breath at rest had disappeared, as had stridor and the use of accessory respiratory muscles. The patient continued with CPAP to maintain patency of the trachea.

Two years after the last dilatation and the start of treatment with CPAP, adherence to continuous positive pressure therapy was considered fine, as evaluated by the compliance recorded on the device, and by interviews with the patient and/or relatives. CPAP is yet maintained throughout the night time. The last bronchoscopy follow-up carried out in May 2017 revealed a sufficiently large trachea and an adequate respiratory space.

\section{Case \#2}

A 69-year-old woman underwent endotracheal intubation due to acute respiratory failure following cardiogenic shock. Few months after discharge she reported effort of breathing at rest, and she presented stridor and use of accessory respiratory muscles, and chest computed tomography (CT) showed the presence of a tracheal stenosis with a residual lumen of $9 \mathrm{~mm}$. The day after the CT scan she was hospitalized at Niguarda Hospital in order to perform an urgent rigid tracheoscopy, which showed a concentric stenosis of about 4-5 mm (Grade III - Myers-Cotton subglottic stenosis classification scale) at the level of the first two cartilaginous rings. A radial section of the trachea was performed with a papillotome and subsequently the stenosis was dilated by means of a balloon $(16 \times 55 \mathrm{~mm}, 4 \mathrm{~atm})$ with restoration of a good canalization.
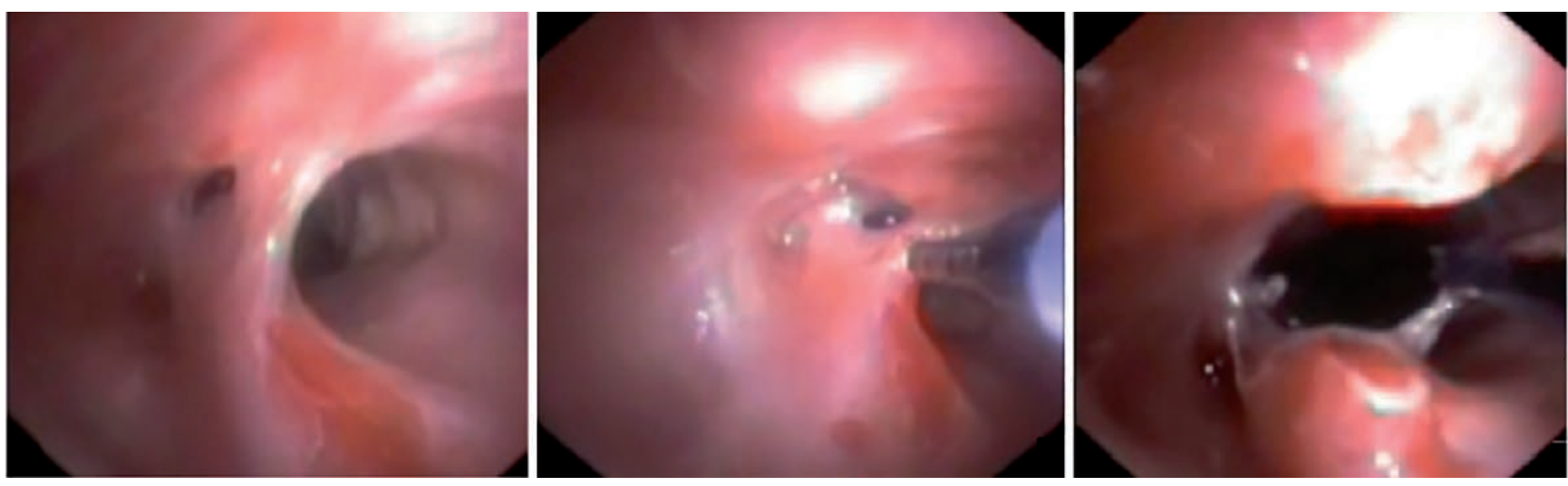

Figure 1. Case \#1. Endoscopic laser surgery. Images represent tracheal stenosis undergone to treatment of precut incision followed by dilation with balloon to obtain an adequate respiratory lumen. 
Downstream of the stenosis, other cartilaginous rings not in axis have been described, as well as the presence of diffuse malacia and granulomatosis of the pars membranacea $2 \mathrm{~cm}$ below the stenosis, determining reduction of the tracheal caliber but with preserved respiratory space. Following the endoscopic intervention performed to recanalize the trachea, the patient started cycles of CPAP ( $\mathrm{PEEP}$ set at $10 \mathrm{cmH}_{2} \mathrm{O}$ ) of two hours in the morning, two hours in the afternoon and all night long, using a single-tube home ventilator (Breas ${ }^{\circledR}$ iSleep 20) with integrated humidifier, with oral-nasal interface (Resmed Quattro FX size M).

She was discharged after three days in good general condition. At home the patient continued with CPAP, held for at least two hours in the afternoon and throughout the night; it was prescribed also aerosol therapy with beclomethasone (1 ampoule 3 times/day).

At the follow-up bronchoscopy performed 13 days after last dilatation, a good tracheal diameter was confirmed, with reduction of the previous granulomatosis and appreciable alignment of the treated segments; however, the malacic component remained yet.

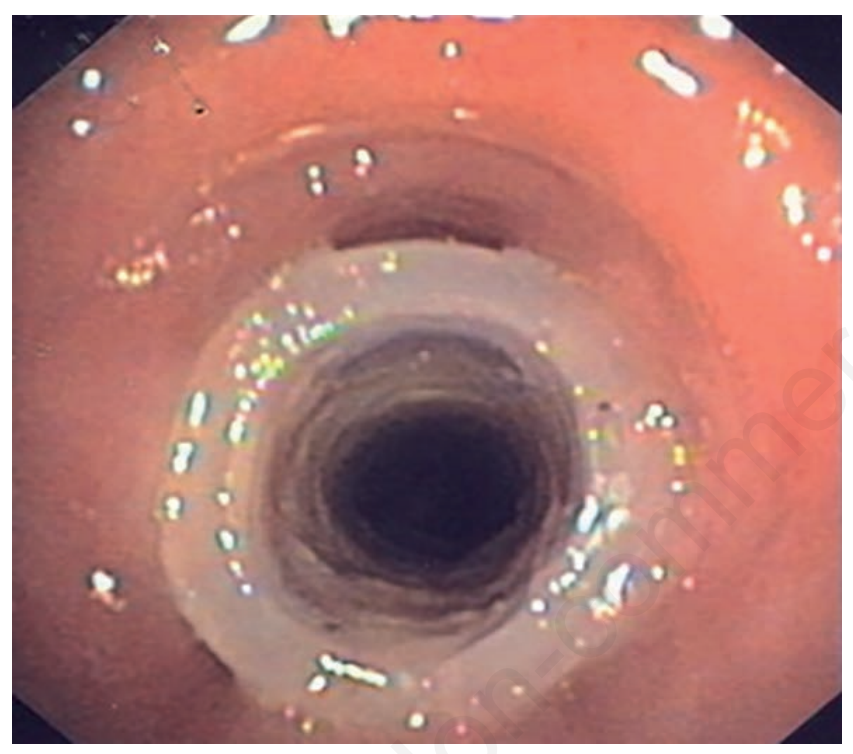

Figure 2. Case \#1. Tracheal dilatation with endoluminal stent. Silicon prosthesis is used to stabilize the tracheal lumen after dilation treatment.
It was recommended to continue CPAP therapy, reducing PEEP to $7.5 \mathrm{~cm} \mathrm{H}_{2} \mathrm{O}$ until the next follow-up.

After three and a half months, the presence of an adequate respiratory space was confirmed at bronchoscopy, without the need for further endoscopic dilations, or presence of respiratory difficulties; the stridor and the use of respiratory muscles disappeared as well. The patient continued for six months a home treatment with CPAP for 1 hour, two times/day, and throughout the night, keeping the previously set pressure unchanged.

\section{Discussion}

Tracheal stenosis is one of the possible long-term complication found both following endotracheal intubation and tracheotomy [5]. The high pressure of the endotracheal tube (or cannula) cuff is recognized as the main cause leading to the stenosis of tracheal lumen. Inflation pressure higher than the capillary perfusion pressure of tracheal mucosa (i.e., $25-35 \mathrm{mmHg}$ ) can cause ischemia, resulting in the ulceration of the mucosa, followed by inflammation, necrosis and partial or complete destruction of the tracheal cartilage [6]. These reactions stimulate the development of scar tissues that obstructs the lumen of the airway, finally resulting in circumferential stenosis [6]. In case of tracheotomy, tracheal stenosis may result from abnormal tissue repair, with excessive granulation tissue formation at the tracheal stoma [2]. Other complications reported with these invasive procedures are sepsis and stoma infection [2,7]. Some known general risk factor such as age and systemic hypotension potentially lead to prolonged intubation length $[2,7]$. Generally, tracheal stenosis may produce no symptoms until the lumen has been reduced by $50-75 \%$. Once the tracheal lumen is $\leq 10 \mathrm{~mm}$, exertional dyspnea occurs; when it becomes $\leq 5 \mathrm{~mm}$, dyspnea at rest or stridor is usually reported [8].

The choice of the best therapeutic approach for the treatment of subglottic tracheal stenosis still represents a challenge. As a matter of fact, laryngotracheal resection is the most considered treatment option [9]. Literature data show that surgical treatment may allow very high success rates at long term with low perioperative morbidity and mortality [9]. During surgery, there is a first phase of resection of the tracheal part - the narrowing - which is followed by the reconstruction of the airway by joining the healthy flaps of the trachea. In addition to surgical techniques, several bronchoscopy procedures have also been used over the years.

These two clinical cases describe an alternative therapeutic strategy to conventional tracheal stenosis therapy, adopting contin-

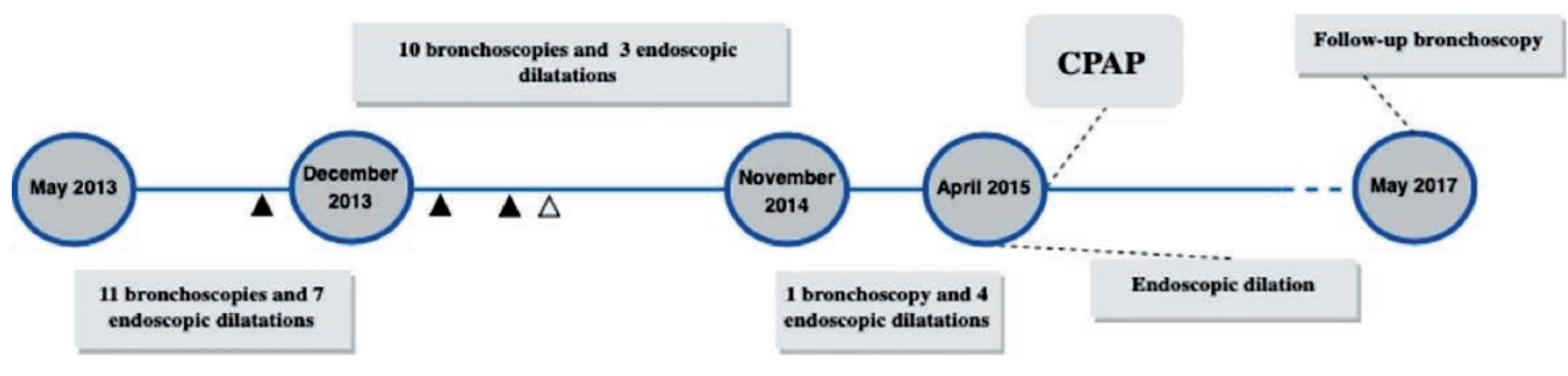

Figure 3. Case \#1 timeline. Black triangle indicates stent placement after endoscopic dilations; unfilled triangle shows stent removal. 
uous positive airway pressure to facilitate the patency of the tracheal diameter previously obtained with endoscopic procedures. The multidisciplinary approach was a key factor, given the skill of respiratory physiotherapists with positive pressure devices and the surgical expertise of thoracic surgeons.

Literature is scanty of other cases in which a similar use of CPAP for the treatment of steno-tracheomalacia (TM) in adulthood has been made [3]; on the contrary, more numerous are the reports showing the effectiveness of this approach in the management of pediatric patients [10]. Continuous positive airway pressure is an effective treatment for infants with moderate-to-severe TM, tracheobronchomalacia (TBM), or bronchomalacia [11]. Bronchoscopy and fluoroscopy have shown that CPAP maintains airway patency during tidal breathing. By essentially creating a pneumatic stent, CPAP prevents the collapse of the airway throughout the respiratory cycle [11]. CPAP applied non-invasively could behave as a pneumatic stent in adult patients with TBM and long-term intermittent treatment may clinically improve those patients who have relapses after conventional endoscopic therapy [12].

Ferguson and Benoist have described three adult subjects, respectively 37, 67 and 66 years old, suffering from chronic respiratory disease and TBM documented by specific signs on spirometric examination (notching on the flow-time curve at the forced vital capacity, FVC) and by the dynamic collapse of the trachea viewed by fibro-bronchoscopy examination [12]. Following the failure of conventional medical therapies, patients underwent intermittent treatment with nasal CPAP. The addition of $10 \mathrm{~cm} \mathrm{H}_{2} \mathrm{O}$ of continuous positive pressure resolved the collapse of the airways during passive expiration and, at the spirometry, the increase in FVC and the disappearance of the notching during forced expiration was observed. Similarly, CPAP has been indicated as a therapeutic strategy to prevent upper airway collapse in a patient with severe tracheomalacia and evident tracheal lumen stenosis [3]. In addition to a better residual functional capacity (FRC), increasing CPAP levels also results in reduced airway collapse. Nevertheless, there is no consensus for adjusting the optimal positive pressure levels. In TBM, CPAP pressures of 7 to $10 \mathrm{~cm} \mathrm{H}_{2} \mathrm{O}$ usually assure airway patency but pressures can be increased by $3 \mathrm{~cm} \mathrm{H}_{2} \mathrm{O}$ incrementally until airway caliber during tidal exhalation is considered satisfactory (e.g., at least $50 \%$ of that noted during inspiration) [13]. In our cases, airway pressure was set at the maximum pressure used to stent the airway according to patient comfort [14].

It is worth mentioning the case report of Wiest et al. on a patient suffering from sleep apnea syndrome, secondary to tracheal stenosis and bilateral paralysis of the recurrent laryngeal nerve. The patient, refusing every type of surgery/endoscopic treatment, was successfully treated by night time application of CPAP at 12 cm $\mathrm{H}_{2} \mathrm{O}$ [15].

Our experience has also produced satisfactory long-term results. In the first case, CPAP allowed to stabilize the trachea after several endoscopic dilatations performed for continuous recurrences of the stenosis, and it was continued at home at $10 \mathrm{cmH}_{2} 0$. In the second case, right after an endoscopic dilatation performed to recanalize the trachea, decision to initiate CPAP therapy was clearly influenced by the positive results obtained with the first case. The patient continued CPAP set at $7.5 \mathrm{~cm} \mathrm{H}_{2} \mathrm{O}$ at home, performed for 1 hour twice a day and during night. Follow-up bronchoscopies, carried out in the first case after two years and in the second case after three months and a half, confirmed the persistence of the tracheal space, thanks to the stabilization of the stenosis, and of an adequate respiratory space.

As far as the interface choice is concerned, Jiang et al. used nasal rather than oral-nasal interfaces as we did. Nasal masks are smaller and associated with a reduction in dead space; certain sideeffects, including abdominal distension resulting from ingesting air, are alleviated; the nasal mask is well tolerated and there is reduced gas leakage, particularly in elderly patients with facial bone malformation [3]. In the two cases described by us, after several attempts with various interface models, both patients chose an oral-nasal interface, perceived more comfortable. The presence of aerophagy only occurred in the first case: this unpleasant issue was overcome by reducing the PEEP level from 12 to $10 \mathrm{~cm} \mathrm{H}_{2} \mathrm{O}$.

In both cases, informed consent was obtained before application of the therapy by a signature on the CPAP administration form.

\section{Conclusions}

CPAP is a relatively simple and non-invasive device to be used with few adverse events. By increasing the endoluminal pressure of the trachea, CPAP behaves as a pneumatic stent, able to improve airflow through the airways, thus preventing any potential collapse. This mechanism unloads the respiratory muscles by reducing the resistance determined by tracheal stenosis, it resolves the shortness of breath and the occurrence of stridor. Although the incidence of tracheal strictures following tracheal intubation or tracheotomy is decreasing, it remains a potential long-term complication that requires a careful multidisciplinary evaluation.

The treatment of tracheal stricture by application of continuous positive pressure represented a valid therapeutic option in the described case series. CPAP therapy stabilized tracheal lumen and, most likely, averted the need of further endoscopic procedures, with a positive potential impact both on direct costs and patients' quality of life. More studies are needed to recommend the use of this technique in a standardized manner.

\section{References}

1. Wain JC. Postintubation Tracheal stenosis. Semin Thorac Cardiovasc Surg 2009;21:284-9.

2. Schönhofer B, Kluge S. Consequences of endotracheal intubation and tracheostomy. In: R.D. Stevens, N. Hart, M.S. Herridgeed, Editors, Textbook of post-ICU medicine: The legacy of critical care. Oxford University Press; 2014.

3. Jiang AG, Gao XY, Lu HY. Diagnosis and management of an elderly patient with severe tracheomalacia : A case report and review of the literature. Exp Ther Med 2013;6:765-8.

4. Boles J-M, Bion J, Connors A, et al. Weaning from mechanical ventilation. Eur Respir J 2007;29:1033-56.

5. Sue RD, Susanto I. Long-term complications of artificial airways. Clin Chest Med 2003;24:457-71.

6. Divatia JV, Bhowmick, K. Complications of endotracheal intubation and other airway management procedures. Indian $\mathrm{J}$ Anesth 2005;49:308-18.

7. Fernandez-Bussy S, Mahajan B, Folch E, et al. Tracheostomy tube placement. J Bronchology Interv Pulmonol 2015;22:357-64.

8. Epstein SK. Late complications of tracheostomy. Respir Care 2005;50:542-9.

9. D'Andrilli A, Venuta F, Rendina EA. Subglottic tracheal stenosis. J Thorac Dis 2016;8:S140-7.

10. Armstrong D. The use of continuous positive airway pressure or non-invasive ventilation as forms of respiratory support in children with cystic fibrosis. Paediatr Respir Res 2013;14:S19-21. 
11. Carden KA, Boiselle PM, Waltz DA, Ernst A. Tracheomalacia and tracheobronchomalacia in children and adults: An in-depth review. Chest 2005;127:984-1005.

12. Ferguson GT, Benoist J. Nasal continuous positive airway pressure in the treatment of tracheobronchomalacia. Am Rev Respir Dis 1993;147:457-61.

13. Murgu S, Colt H. Tracheobronchomalacia and excessive dynamic airway collapse. Clin Chest Med 2013;34: 527-55.
14. van der Schans CP, van der Mark TW, de Vries G, et al. Effect of positive expiratory pressure breathing in patients with cystic fibrosis. Thorax 1991;46:252-6.

15. Wiest GH, Ficker JH, Lehnert G, Hahn EG. [Secondary obstructive sleep apnea syndrome in a patient with tracheal stenosis and bilateral recurrent paresis. Successful treatment with nasal continuous positive airway pressure therapy].[Article in German]. Dtsch Med Wochenschr 1998; 123:522-6. 